\title{
Development and Validation of HPTLC Method for Simultaneous Estimation of Metolazone and Spironolactone in Bulk Drug and Pharmaceutical Dosage Form
}

\author{
Celina Nazareth ${ }^{1 *}$, B. Shivakumar ${ }^{2}$, Prasad Reddy ${ }^{3}$ and B.M. Gurupadayya ${ }^{4}$ \\ ${ }^{1 *}$ PES's Rajaram and Tarabai Bandekar College of Pharmacy, Farmagudi, Goa, India. \\ ${ }^{2}$ BLDEA College of Pharmacy, Bijapur, India. \\ ${ }^{3}$ Samskruti College of Pharmacy, Kondapur, Hyderabad, India. \\ ${ }^{4}$ JSS College of Pharmacy, JSS University, Mysore, India.
}

\begin{abstract}
A simple, precise and accurate HPTLC method has been developed for the simultaneous analysis of Metolazone and Spironolactone in bulk drugs and tablet formulation. Chromatographic separation was carried out on Merck HPTLC aluminium plates of Silica gel $60 F_{254}$, using n-propanol : triethylamine (7:3 $v / v)$ as the mobile phase. HPTLC separation of the two drugs followed by densitometric measurement was performed in the absorbance mode at $240 \mathrm{~nm}$. The drugs were resolved with $R_{f}$ values of 0.60 and 0.69 for Metolazone and Spironolactone respectively. The linear regression analysis data for the calibration plots showed good linear relationship with $r^{2}$ value 0.99886 and 0.99862 for Metolazone and Spironolactone, respectively in the concentration range of $300-700 \mathrm{ng} / \mathrm{spot}$ for Metolazone and 300-700 $\mathrm{ng} / \mathrm{spot}$ for Spironolactone. The method was validated following the ICH guidelines in terms of accuracy, precision, specificity and robustness. The limit of detection and quantitation were 200 and $600 \mathrm{ng} / \mathrm{spot}$ respectively for Metolazone and 200 and $600 \mathrm{ng} / \mathrm{spot}$ for Spironolactone. The proposed developed and validated HPTLC method can be utilised for identification and quantitative analysis of Metolazone and Spironolactone in bulk drugs and pharmaceutical dosage form.
\end{abstract}

Keywords: HPTLC, Metolazone, Spironolactone, Validation.

\section{INTRODUCTION}

Metolazone is an oral diuretic agent commonly classified with the thiazide diuretics. Chemically it is 7-chloro-1, 2, 3, 4-tetrahydro-2-methyl-4-oxo-3-o-tolyl-6-quinazoline sulphonamide (Fig.1.1). A quinazoline-sulfonamide is considered a thiazide-like diuretic which is long-acting, so useful in chronic renal failure. It is primarily used to treat congestive heart failure and high blood pressure. Metolazone indirectly decreases the amount of water reabsorbed into the bloodstream by the kidney, so that blood volume decreases and urine volume increases. This lowers blood pressure and prevents excess fluid accumulation in heart failure [1-5]

Spironolactone is a potassium sparing diuretic agent. Chemically it is $7 \alpha$-acetyl thio-3-oxo-17 $\alpha$ pregn-4-ene-21, $17 \beta$-carbo-lactone (Fig.1.2). It is an aldosterone antagonist chemically related to the mineralocorticoid aldosterone. It antagonizes potassium loss induced by other diuretics and slightly adds to their natriuretic effect. It is used mainly in the treatment of refractory oedema in patients with congestive heart failure, nephrotic syndrome, or hepatic cirrhosis and as adjuvant to thiazide to prevent hypokalaemia [1-6].

Drugs in combination are increasingly used nowadays due to advantages of increased potency, multiple action, greater patient compliance, decreased side effects and faster relief. High Performance Thin Layer Chromatography i.e. HPTLC is routinely used in analysis of pharmaceutical formulations in finger print profiling and quantification as well as impurity profiling and stability studies. These methods offer several advantages over HPLC as being simple, rapid cost effective, with increased sample throughput and the need for minimum sample preparation. The greatest advantage of HPTLC over HPLC is that several samples can be run simultaneously using a small quantity of mobile phase thus reducing the analysis time and cost per analysis $[8,9]$.

Literature survey revealed that a number of analytical methods like UV [10-14], RP-HPLC [15-20], HPTLC [21-22] have been reported for the estimation of Metolazone and Spironolactone as individual and in combination with other drugs. However, no HPTLC method has been reported for the simultaneous estimation of Metolazone and Spironolactone in their combined tablet dosage form. Hence, to capitalise on the benefits of HPTLC methods, the present work aims to develop and validate a HPTLC method for the simultaneous estimation of Metolazone and Spironolactone in combination. 
<smiles>Cc1ccccc1N1C(=O)c2cc(S(N)(=O)=O)c(Cl)cc2NC1C</smiles>

(1)<smiles>CC(=O)S[C@H]1CC2=CC(=O)CC[C@]2(C)[C@H]2CC[C@]3(C)[C@@H](CC[C@]34CCC(=O)O4)[C@H]12</smiles>

(2)

Fig. 1 Structures of (1) Metolazone and (2) Spironolactone

\subsection{Chemicals and reagents}

\section{MATERIALS AND METHODS}

All chemicals and reagents used were of AR grade and Silica gel $60 \mathrm{~F}_{254}$ with thickness $200 \mu \mathrm{m}, \mathrm{E}-$ Merck, Germany was used as stationary phase. Metolazone and Spironolactone were supplied as gift samples, by Centaur pharmaceuticals Pvt. Ltd., Tivim, Goa. Metolactone 2.5 tablet containing $2.5 \mathrm{mg}$ Metolazone and 50 $\mathrm{mg}$ Spironolactone were obtained commercially from a local pharmacy.

\subsection{HPTLC instrument and Chromatographic conditions}

The instrument utilised for analysis was CAMAG-HPTLC System comprising of CAMAGLINOMAT-V sample applicator, CAMAG TLC-Scanner 3 controlled by win CATS software (version 1.4.6), CAMAG-UV cabinet and CAMAG twin trough glass chamber with stainless steel lids. The detector used was a Deuterium lamp emitting a continuous UV spectrum between $190-400 \mathrm{~nm}$.

Chromatographic separation was achieved on Aluminium foil plates coated with 0.2-mm layer of Silica gel $60 \mathrm{~F}_{254}$ (Merck). Samples were applied to the plates, as 8-mm bands, by means of a Camag Linomat V sample applicator used at a constant application rate of $150 \mathrm{nl} / \mathrm{s}$. Plates were developed using ascending mode of chromatographic separation with n- Propanol: Triethylamine 7:3 $(v / v)$ as mobile phase in a Camag twin-trough chamber previously saturated with mobile phase vapours for $20 \mathrm{~min}$ at room temperature $\left(25 \pm 2^{\circ} \mathrm{C}\right)$. The development distance was approximately $80 \mathrm{~mm}$. After development, the plates were dried and densitometric scanning was done in absorbance mode at $240 \mathrm{~nm}$ by use of a Camag TLC Scanner 3. The slit dimensions were $6 \mathrm{~mm} \times 0.45 \mathrm{~mm}$.

\subsection{Preparation of standard and sample solutions}

A standard stock solution of Metolazone was prepared by accurately weighing Metolazone $(10 \mathrm{mg})$ into a 10-ml volumetric flask. The drug was dissolved in methanol and the solution was diluted to volume. From this one $\mathrm{ml}$ of the solution was further diluted to $10 \mathrm{ml}$ using methanol. So also, a standard stock solution of Spironolactone was prepared by accurately weighing Spironolactone $(10 \mathrm{mg})$ into a $10-\mathrm{ml}$ volumetric flask. The drug was dissolved in methanol and the solution was diluted to volume. From this one $\mathrm{ml}$ was further diluted to $10 \mathrm{~mL}$ using methanol.

Twenty tablets of the pharmaceutical formulation Metolactone 2.5 (containing $2.5 \mathrm{mg}$ Metolazone and $50 \mathrm{mg}$ Spironolactone) were assayed. They were crushed to a fine powder and an amount of the powder corresponding to approximately $2.5 \mathrm{mg}$ Metolazone and $50 \mathrm{mg}$ Spironolactone was weighed and transferred into a $10 \mathrm{ml}$ volumetric flask. After addition of $5 \mathrm{ml}$ methanol and sonication (15 mins), the solution was diluted to volume with methanol and filtered through a Whatman filter paper. This solution was used for Metolazone while for Spironolactone; one $\mathrm{ml}$ was further diluted to $20 \mathrm{ml}$ and used.

\subsection{Sample analysis}

Using appropriate dilutions of standard stock solutions, $5.0 \mu \mathrm{l}$ of the two drug standards was applied to a TLC plate. The analysis was repeated five times, followed by development and scanning at $240 \mathrm{~nm}$. The chromatogram obtained of standard mixture of Metolazone and Spironolactone is shown in Fig. 2. 


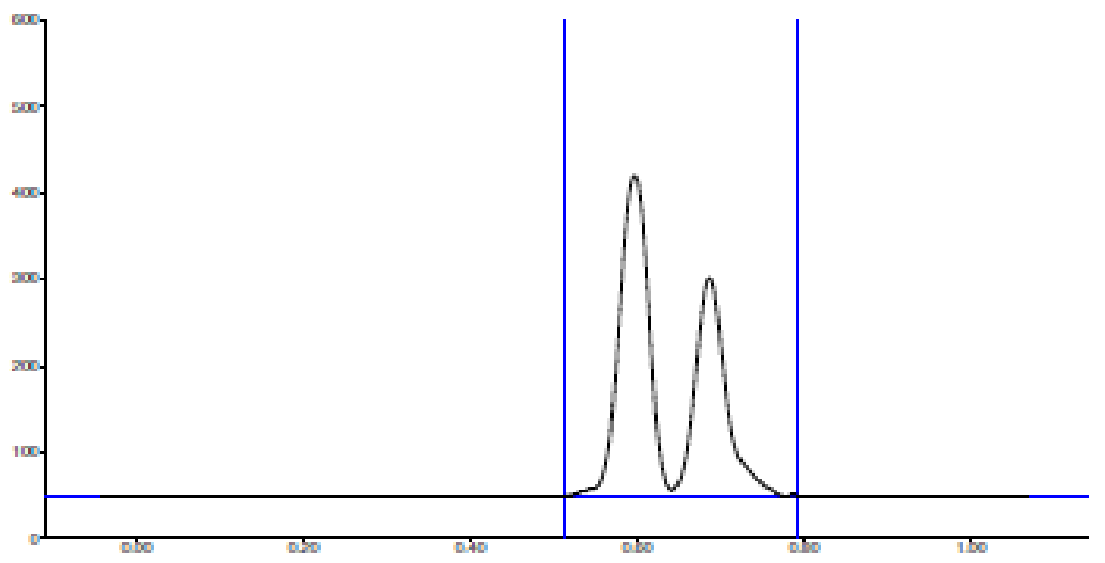

Fig.2 Chromatogram of Standard Mixture

\section{RESULTS AND DISCUSSION}

\subsection{Method Development}

In preliminary attempts separation of the two drugs was tried with the following mobile phases:

Trial 1. Toluene: Acetonitrile: glacial Acetic acid $(7: 3: 0.2 \mathrm{v} / \mathrm{v} / \mathrm{v})$

Trial 2. n-Hexane: Ethyl acetate: glacial Acetic acid ( 5:5:0.05 v/v/v)

Trial 3. Ethyl acetate: Chloroform: Formic acid: Triethylamine (17:3:0.1:0.1 v/v/v/v)

Trial 4. n-Hexane: Ethyl acetate: Methanol: Water: glacial Acetic acid ( 4:8:3:0.4:0.2 v/v/v/v/v)

However both drugs would travel along with the mobile phase front with no separation. Finally, separation of the two drugs was achieved by employing the mobile phase of $n$-Propanol : Triethylamine (7:3 v/v).

Analysis of the drug samples was performed by applying $5 \mu 1$ of the appropriate dilutions of standard stock solutions to a HPTLC plate. The analysis was repeated five times, followed by development and densitometric scanning at $240 \mathrm{~nm}$. The chromatogram obtained of the standard mixture of Metolazone and Spironolactone is shown in Fig.2. The drugs were resolved with $\mathrm{R}_{\mathrm{f}}$ values of 0.60 and 0.69 for Metolazone and Spironolactone respectively.

\subsection{Method Validation}

The proposed method was validated as per ICH guidelines with respect to linearity, accuracy, precision, LOD, LOQ, specificity and robustness [23].

\subsubsection{Linearity}

The linearity of an analytical method is its ability to elicit test results that are directly proportional to the concentration of analyte in sample within a given range. From the diluted standard solution $100 \mu \mathrm{g} / \mathrm{ml}$ of Metolazone, 2 to $8 \mu$ l solutions were spotted on HPTLC plate to obtain final concentration 200-800 ng/spot for Metolazone. Similarly in case of $100 \mu \mathrm{g} / \mathrm{ml}$ of Spironolactone, 2 to $8 \mu$ l solutions were spotted on HPTLC plate to obtain final concentration 200-800 ng/spot for Spironolactone. The plate was then developed as per the procedure and scanned densitometrically. The linear regression analysis data for the calibration plots showed good linear relationship with $\mathrm{r}^{2}$ value 0.99886 and 0.99862 for Metolazone and Spironolactone, respectively in the concentration range of $300-700 \mathrm{ng} / \mathrm{spot}$ for Metolazone and 300-700 ng/spot for Spironolactone. The linearity overlain chromatograms for both drugs are given in Fig. 3. The calibration curves for both drugs are given in Fig. 4. 


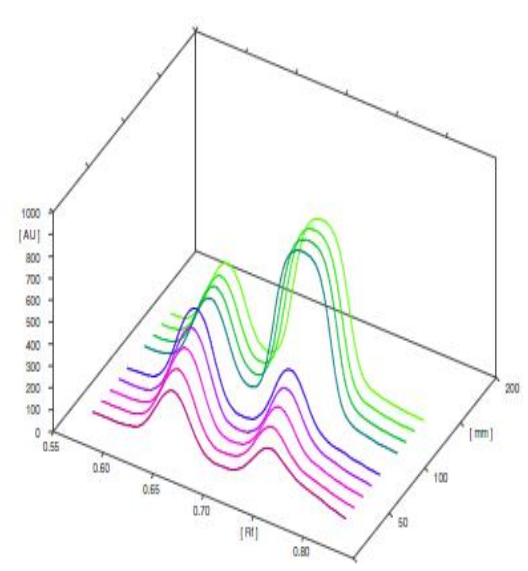

(1) Metolazone

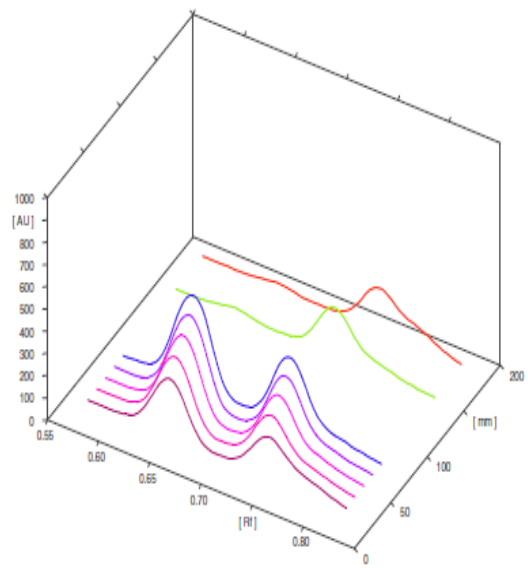

(2) Spironolactone

Fig.3 Linearity overlain Chromatogram of (1) Metolazone and (2) Spironolactone

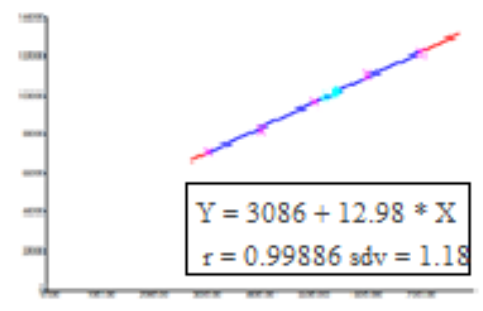

(1) Metolazone

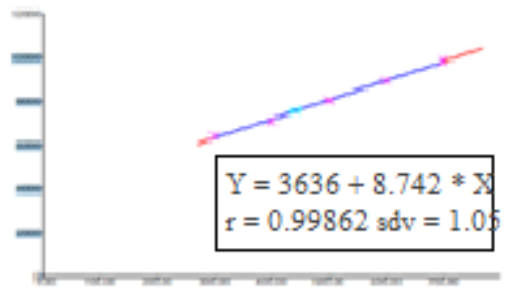

(2) Spironolactone

Fig.4. Calibration curves of (1) Metolazone and (2) Spironolactone

\subsubsection{Accuracy}

Accuracy may be expressed as percentage recovery. It was determined by applying the method to drug sample (tablet powder) to which a known amount of Metolazone and Spironolactone standard corresponding to 80,100 and $120 \%$ of label claim had been added. The experiment was conducted in triplicate. Analysis was done by running the chromatogram in optimised mobile phase. The mean percent recoveries of the drugs at different concentration levels are reported in Table 1. The results imply that the developed method was accurate for the determination of the drugs.

\subsubsection{Precision}

The precision of an analytical method is the degree of agreement among individual test results when the method is applied repeatedly to multiple samplings of homogenous samples. Intraday and Interday precision of the method was verified by repeatability and intermediate precision studies. The precision of the method was expressed as CV\% i.e. percent coefficient of variance. The results of precision studies as listed in Table 1 show that, the method is precise.

\subsubsection{Limit of detection (LOD) and Limit of Quantitation (LOQ)}

The detection limit is defined as the lowest concentration of an analyte that can be detected under the analytical conditions to be used. Here the presence of analyte can be seen but its concentration cannot be quantitatively measured. The quantitation limit is the lowest concentration that can be determined with acceptable accuracy and precision under the analytical conditions. Generally, the LOQ can be estimated as three times that of LOD. 
The limit of detection and quantitation were 200 and $600 \mathrm{ng} / \mathrm{spot}$ respectively for Metolazone and 200 and $600 \mathrm{ng} / \mathrm{spot}$ respectively for Spironolactone. This indicates the method is sufficiently sensitive.

\subsubsection{Specificity}

Specificity is the ability to assess unequivocally the analyte in the presence of components that may be expected to be present. The specificity of the method was ascertained by analysis of drug standards and tablet formulation in presence of the diluents and the mobile phase. The mobile phase resolved both the drugs. The identities of the bands for Metolazone and Spironolactone were confirmed by comparing the $\mathrm{R}_{\mathrm{f}}$ values and spectra of the bands with those of standards as shown in Fig.5. There were no interferences from the mobile phase and diluent.

metalazone on all Tracks

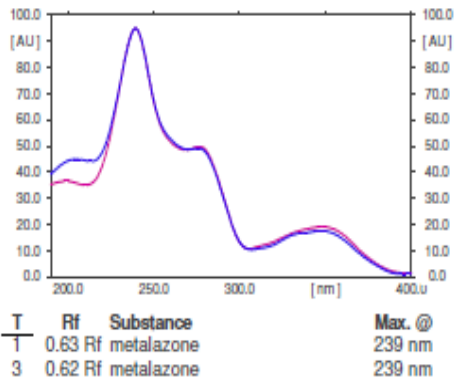

(1) Metolazone spirinolactone on all Tracks

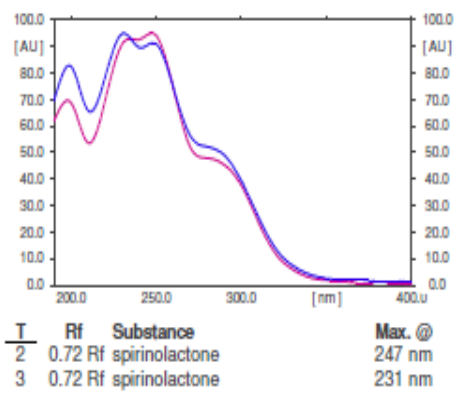

(2) Spironolactone

Fig.5 Specificity (1) Metolazone and (2) Spironolactone

\subsubsection{Robustness}

The robustness was studied by evaluating the effect of small but deliberate changes in chromatographic conditions (saturation time of chamber $10 \mathrm{~min}$ ), change in mobile phase composition [ $\mathrm{n}$ - Propanol : Triethylamine 8:2 $(v / v)$ ] and change of analyst. The CV\% values as given in Table 1 show that the method is robust.

Table 1 Summary of Validation Parameters

\begin{tabular}{|l|c|c|}
\hline \multicolumn{1}{|c|}{ Parameters } & \multicolumn{2}{|c|}{ Results } \\
\cline { 2 - 3 } & Metolazone & Spironolactone \\
\hline Linearity Range (ng/spot) & $300-700$ & $\mathrm{Y}=300-700$ \\
\hline Regression Equation (n=5) & $\mathrm{Y}=3086+12.98 * \mathrm{X}$ & 0.99862 \\
\hline Correlation coefficient (r ${ }^{2}$ ) & 0.99886 & \\
\hline Accuracy (mean \% recovery) & $98.3 \%$ & $89.2 \%$ \\
At 80\% & $100.2 \%$ & $88.3 \%$ \\
At 100\% & $106.9 \%$ & $90.4 \%$ \\
At 120\% & & \\
\hline Precision ( CV\% ) & 1.971 & 0.209 \\
Intraday precision (n=3) & 1.634 & 2.232 \\
Interday precision (n=3) & & $200 \mathrm{ng}$ \\
\hline Limit of detection (LOD) & $200 \mathrm{ng}$ & $600 \mathrm{ng}$ \\
\hline Limit of Quantitation (LOQ) & $600 \mathrm{ng}$ & 2.589 \\
\hline Robustness (CV \%) & 2.596 & $88.1 \%$ \\
\hline Mean \% Assay & $106.8 \%$ & \\
\hline
\end{tabular}

\subsection{Assay of tablet}

Twenty tablets of the pharmaceutical formulation Metolactone 2.5 (containing $2.5 \mathrm{mg}$ Metolazone and $50 \mathrm{mg}$ Spironolactone) were assayed using the developed method. Satisfactory results were obtained as the mean concentration found was in good agreement with the label claimed. The results tabulated in Table 2 showed that the developed method could be used for performing the assay of the drugs in marketed formulations. 
Table 2 Analysis of marketed formulation

\begin{tabular}{|c|c|c|c|c|}
\hline \multirow{2}{*}{ Tablet } & Components & $\begin{array}{c}\text { Concentration } \\
(\mathbf{n g} / \mathbf{s p o t})\end{array}$ & $\begin{array}{c}\text { Concentration } \\
\text { found (ng/spot) } \\
(\mathbf{n}=\mathbf{3})\end{array}$ & Mean \% recovery \\
\hline \multirow{2}{*}{ Metolactone 2.5 } & Metolazone & 500 & 534 & $106.8 \%$ \\
\cline { 2 - 5 } & Spironolactone & 500 & 440.97 & $88.1 \%$ \\
\hline
\end{tabular}

\section{CONCLUSION}

The developed High Performance Thin Layer Chromatographic method is accurate, precise, robust and specific, with no interferences from excipients. It can be readily adopted for the selective analysis of Metolazone and Spironolactone in pharmaceutical formulations without the need for prior separation. The method could be employed for the quality control of the active pharmaceutical ingredients in routine analysis as well as for the simultaneous assay of drugs in a combined tablet formulation. The developed method offers a cost effective alternative to HPLC separation of the two drugs in combination.

\section{ACKNOWLEDGEMENTS}

The authors are thankful to Centaur pharmaceuticals Pvt. Ltd., Tivim, Goa for the gift samples of pure Metolazone and Spironolactone. The authors would also like to thank Anchrom Enterprises Pvt. Ltd, MulundMumbai for providing the facilities for carrying out this research work.

\section{REFERENCES}

[1] M.J. O’Neil, The Merck Index (fifteenth ed., RSC publishing, 2013), pp. 6222, 8885

[2] United States Pharmacopoeia (Vol III, thirty fourth ed., , Rockville, MD: The United States Pharmacopeial Convention, 2011), 3506, 4267-4268.

[3] British Pharmacopoeia (Vol II, The Stationary Office on behalf of MHRA, 151 Buckingham Palace Road, London, 2012), 1458-1459, 2030-2032.

[4] M.C. Anthony, M. David Osselton, Clark's analysis of drugs and poison (Vol II, third ed.), 313, 1273-1568.

[5] K.D. Tripathi, Essentials of Medical Pharmacology (sixth ed., Jaypee Brothers Medical Publishers (P) ltd. 2008), 561-578.

[6] Indian Pharmacopoeia (Vol III, The Indian Pharmacopoeia Commission, Ghaziabad, Govt. of India Ministry of Health and Family Welfare, 2010), 2147-2148.

[7] http://www.pubchem.ncbi.nlm.nih.gov (October 2013)

[8] P.D. Sethi, D. Charegaonkar, Identification of Drugs in Pharmaceutical Formulations by Thin Layer Chromatography (second ed., CBS Publishers, 2008), 1-20.

[9] P.D. Sethi, Sethi's HPTLC (Kongposh Publications Pvt. Ltd., 2011), 9-18.

[10] S. Sandeepkumar, S. Manjunath, A. Raju, V.S. Chouhan, Ultra violet and derivative spectrophotometric methods for estimation of Metolazone in pharmaceuticals, Inter J Pharma Sci, 2(3,) 2011, 204-209.

[11] S. Sandeepkumar, S. Manjunath, A. Raju, V.S. Chouhan, Development and Validation of Visible Spectrophotometric methods for the Estimation of Metolazone in Pharmaceutical Dosage Forms, Der Pharma Chemica, 3(2) ,2011, 512-516.

[12] B.J. Chandra , S.G. Siven, C. Manikandan, Simultaneous spectrophotometric methods for the estimation of Torasemide and Spironolactone in tablet dosage form, J Anal Chem, 1(1), 2010, 1-5.

[13] A. Chaudhary, K.R. Vadalia, P. Thummer, Development and validation of ratio derivative spectrophotometric for simultaneous estimation of metolazone and spironolactone in pharmaceutical dosage form, IJPSR, 3(10), 2012, 3999-4003.

[14] B. Umadevi, T. Vetrichelvan, Simultaneous estimation of Metolazone and Spironolactone in combined tablet Dosage Form by UV Spectroscopy, Int.J. Pharm Tech Res, 3(4), 2011, 2068-2074.

[15] L.R. Khanchandani, K.K. Bhatt, D.A. Shah, U.K. Chhalotiya, Development and validation of liquid chromatographic method for simultaneous estimation of Spironolactone and Hydroflumethiazide in pharmaceutical dosage form, Novus International Journal of Chemistry, 2(1), 2013, 13-19.

[16] V. Chandrakanth, V. Tallada, Development and Validation of RP-HPLC Method for Simultaneous Estimation of Spironolactone and Frusemide in Bulk and Pharmaceutical Dosage Forms, JPR, BioMedRx, 5(8), 2012, 3998-4000.

[17] M.I. Walash, N. El-Enany, M.I. Eid, M.E. Fathy, Simultaneous determination of metolazone and spironolactone in raw materials, combined tablets and human urine by high performance liquid chromatography, Anal. Methods, 5, 2013, 5644-5656.

[18] M.A. Hegazy, F.H. Metwaly, M. Abdelkawy, N. S. Abdelwahab, Validated Chromatographic Methods for Determination of Hydrochlorothiazide and Spironolactone in Pharmaceutical Formulation in Presence of Impurities and Degradants, J Chromatogr Sci, 49 (2), 2011, 129-135.

[19] V. Jadhav, P. Mande, V. Kadam, Validation of Reverse Phase High Performance Liquid Chromatography Method of Metolazone and its Determination in Bulk Drug and Pharmaceutical Dosage Form, J Pharma Res, 2(5), 2009, 961-963.

[20] D. Jain, A. Acharya, V. Jain, S. Bhardwaj, R. Laxman, Development and Validation of RP-HPLC Method For Simultaneous Determination of Spironolactone and Torsemide In Pharmaceutical Dosage Form, Inter J Res Ayu Pharma, 1 (2), $2010,459-467$.

[21] R. Dubey, V.K. Bhusari, S.R. Dhaneswar, Validated HPTLC Method for Simultaneous Estimation of Losartan potassium and Metolazone in Bulk Drug and Formulation, Der Pharmacia Lettre, 3(2), 2011, 334-342.

[22] J.A. Wayadande, R. Dubey, V.K. Bhusari, S.R. Dhaneshwar, Validated HPTLC Method for Simultaneous Estimation of Ramipril and Metolazone in Bulk Drug and Formulation, Der Pharmacia Sinica, 2(4), 2011, 286-294.

[23] Q2B Validation of Analytical Procedures,

http://www.ich.org/fileadmin/Public_Web_Site/ICH_Products/Guidelines/Quality/Q2_R1/Step4/Q2_R1__Guideline.pdf (15 October 2013). 